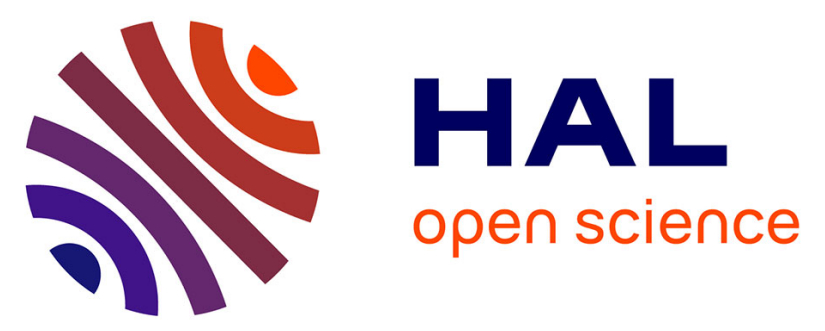

\title{
New Control Plane in 3GPP LTE/EPC Architecture for On-Demand Connectivity Service
}

\author{
Siwar Ben Hadj Said, Malla Reddy Sama, Karine Guillouard, Lucian Suciu, \\ Gwendal Simon, Xavier Lagrange, Jean-Marie Bonnin
}

\section{- To cite this version:}

Siwar Ben Hadj Said, Malla Reddy Sama, Karine Guillouard, Lucian Suciu, Gwendal Simon, et al.. New Control Plane in 3GPP LTE/EPC Architecture for On-Demand Connectivity Service. CLOUDNET 2013: 2nd IEEE International Conference on Cloud Networking, Nov 2013, San Francisco, United States. hal-00876149

\section{HAL Id: hal-00876149 \\ https://hal.science/hal-00876149}

Submitted on 23 Oct 2013

HAL is a multi-disciplinary open access archive for the deposit and dissemination of scientific research documents, whether they are published or not. The documents may come from teaching and research institutions in France or abroad, or from public or private research centers.
L'archive ouverte pluridisciplinaire HAL, est destinée au dépôt et à la diffusion de documents scientifiques de niveau recherche, publiés ou non, émanant des établissements d'enseignement et de recherche français ou étrangers, des laboratoires publics ou privés. 


\title{
New Control Plane in 3GPP LTE/EPC Architecture for On-Demand Connectivity Service
}

\author{
Siwar Ben Hadj Said*, Malla Reddy Sama*, Karine Guillouard*, Lucian Suciu* \\ Gwendal Simon $^{\dagger}$, Xavier Lagrange ${ }^{\dagger}$ and Jean-Marie Bonnin ${ }^{\dagger}$ \\ *Orange Labs, France †Télécom Bretagne, France \\ Emails: \{siwar.benhadjsaid, mallareddy.sama, karine.guillouard, lucian.suciu\}@orange.com \\ Emails: \{gwendal.simon, xavier.lagrange, jm.bonnin\}@telecom-bretagne.eu
}

\begin{abstract}
The on-demand connectivity service is one of the main requirements of the cellular data network. It consists in moving sessions transparently and temporarily from one network equipment to another without causing user session interruption. This service enables networks to cope with the ever-changing network condition such as sudden congestion or arbitrary network equipment failure. In this paper, we argue that the cellular data networks such as LTE/EPC lack the network visibility and control elasticity that enable the on-demand connectivity service. The Software Defined Networking (SDN) is an emerging trend that should be considered to overcomes the above drawback. As a first step, we propose an OpenFlow-based control plane for LTE/EPC architectures. Using resiliency and load balancing use cases, we show that our proposal guarantees the on-demand connectivity service.
\end{abstract}

Index Terms-SDN; OpenFlow; 3GPP; LTE; EPC architecture

\section{INTRODUCTION}

According to analysts [1], mobile data traffic will continue growing significantly until 2017. On the other hand, a study [2] recently highlighted that network operators risk an "end of profit" sometime before mid 2015. Indeed, the total network cost should exceed the total revenue very soon. One of the main causes is the existing architecture of Long Term Evolution (LTE)/Evolved Packet Core (EPC) network. The LTE/EPC architecture has been designed to provide seamless IP connectivity between user equipment (UE) and external Packet Data Networks (PDNs). Today's LTE/EPC architecture experiences a period of rapid and massive change due to dynamic and unpredictable traffic patterns [3]. In the light of these predictions, network operators are invited to revisit the design and capabilities of their architectures with a twofold objective of reducing expenses and introducing new revenue generating services.

One of the main requirements of LTE/EPC is to ensure on-demand connectivity service. This service consists in moving active sessions transparently and temporarily from one network equipment to another without causing user session interruption. On-demand connectivity service is especially critical in situations such as network equipment failure, overload situations and during energy saving measures. In this paper, we focus on two key aspects of on-demand connectivity services, which are $(i)$ resiliency, i.e. restoring active sessions when one critical equipment fails, and (ii) load-balancing, i.e. spreading the load across multiple network equipments and links during traffic peaks. Any proposal for a new LTE/EPC network architecture should address both requirements.

Several recent papers [4]-[7] have proposed evolutions of EPC architectures with the goal of coping with mobile data traffic explosion and ensuring on-demand connectivity. For instance, in [8], authors proposed to move the MME functions, the control plane of the SGW (SGW-C) and the PGW (PGW-C) into separated virtual machines and lifted up to the cloud. They replaced the data plane of the EPC core by OpenFlow switches. The same standard 3GPP interfaces are still used between these control entities. In [9], contributors proposed to split the SGW and PGW functionalities in a new way. The intelligence and decision making is centralized in the combined S/P-GW controller. The packet forwarding function is distributed among OpenFlow switches. In [10], the authors proposed a SDN controller for cellular networks (CellSDN) which is logically centralized and realizes highlevel service police by directing traffic through a sequence of data plane nodes. Today, the network equipment reconfiguration or upgrade is a cumbersome task as it requires the use of Command Line Interface (CLI) or other vendor-specific network management tools. In other words, such network upgrade require each manufacturer to allow reconfiguration of thousands of network equipments.

In this paper, we propose to revisit LTE/EPC in a more radical way by integrating Software Defined Networking (SDN) principles to the EPC network. SDN is a recent trend in communications networking, whereby the behavior of network equipments can be specified and controlled from a single, high-level software program. This trend is reshaping the way networks are designed, managed, and secured. SDN replaces manual interface of the network equipment with a programmatic interface, which enables the automation of tasks such as configuration and policy management [11]. OpenFlow (OF) is one of the main protocols that apply the SDN concepts (in southbound interface [12]) as it enables the remote softwarebased control and management of network equipments with open interfaces in the data plane. Indeed, opening up interfaces to program the network equipments makes the network control, upgrade and management easier. Moreover, the network can be easily extended with new functionalities.

Our contributions are as follows. We first recall the main weaknesses of LTE/EPC architectures. In the line with SDN 
concept, we then propose a new control plane, which is based on the OF protocol. We then show the benefits one can expect from such OF-based LTE/EPC architecture. Our focus in this paper is resiliency and load-balancing. We show that OFbased implementation enables efficient implementation of both key aspects of on-demand connectivity service. Although our proposal can appear appealing from a theoretical standpoint, we do not hide that such shift is not a trivial task. We discuss some of the most prominent implementation issues that prevent fast deployment of SDN-based EPC in today's network. With this in mind, we conclude this paper by some future works.

\section{BACKGROUND}

\section{A. LTE/EPC architecture}

The LTE/EPC architecture is composed of the Evolved Universal Terrestrial Radio Access Network (E-UTRAN) and the EPC core as shown in Fig. 1. The E-UTRAN includes base stations, which are called eNodeBs (eNBs). The EPC consists in four network elements namely Serving Gateway (SGW), PDN Gateway (PGW), and Mobility Management Entity (MME) [13]. The GPRS Tunneling Protocol (GTP) is the main communication protocol within the LTE/EPC architecture.

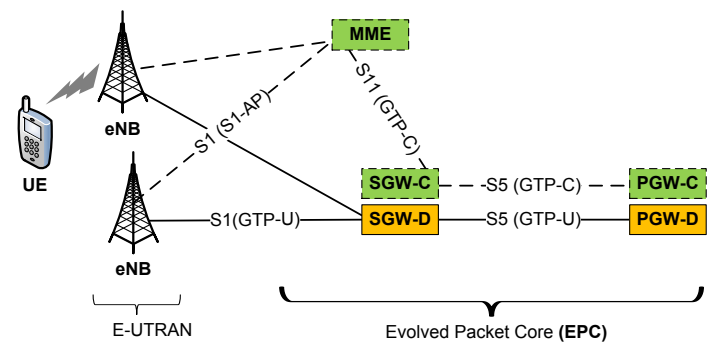

Fig. 1. LTE/EPC architecture.

\section{B. Drawbacks}

The initial attachment and the data plane establishment are the main session management procedures in LTE/EPC networks [13]. The major drawbacks of these procedures are:

- The initial attachments lead to a systematic establishment of the data plane (i.e. GTP tunnel between eNB, SGW and PGW) even when there is no data traffic to be sent.

- The data plane establishment procedure is unaware of the session type. For instance, the UE inactivity timer is locally pre-configured in the eNB and has static value.

- The GTP tunnel is identified in each node with a Tunnel Endpoint Identifier (TEID), an IP address and a UDP port number [14]. The TEID values are locally allocated by each node. Therefore, new TEID values should be exchanged for each node relocation. This challenges the network elasticity.

To address the above issues, we propose to reshape the LTE/EPC architecture by introducing the OF protocol.

\section{OF-BASED LTE/EPC ARCHITECTURE}

\section{A. Architecture Description}

To enable the on-demand connectivity service, we propose a new control plane for the LTE/EPC architecture. In our proposal, we replace the control protocols that run on the S1MME (between MME and eNB) and the S11 (between MME and SGW) interfaces by the OF protocol as shown in Fig. 2.

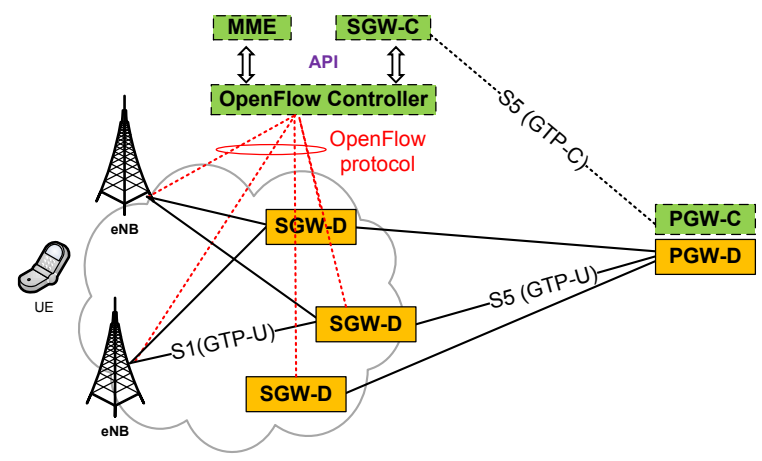

Fig. 2. OF-based LTE/EPC architecture.

In the line with the SDN principle, we propose to separate out all control functions from the data forwarding function in SGWs of the same pool area. As a result, the whole intelligence in the SGW (SGW-C software) and MME is centralized and runs on top of the OpenFlow Controller (OF-ctr) as an application. The data forwarding function is performed by the SGW data plane (SGW-D). The architecture is composed of the following entities:

OpenFlow Controller (OF-ctr): is the main component of our architecture as it manages the forwarding plane of eNB and SGW-D. The OF-ctr is responsible for user session establishment and load monitoring at the data plane.

$M M E$ : is responsible for UE authentication and authorization, and intra-3GPP mobility management. In our architecture, the $\mathrm{MME}$ is no more responsible for the SGW and PGW selection. The MME communicates with the OF-ctr using Application Programming Interface (API). The 3GPP interface between the MME and HSS is still maintained.

SGW control plane $(S G W-C)$ : represents the SGW's intelligence part. It is responsible for GTP tunnel establishment including TEIDs allocation. The SGW-C allocates unique TEID value per session for the uplink traffic within the S1-U interface. It allocates also unique TEID value for the downlink traffic within S5-U interface. With the OF protocol, the Of-ctr can set counters in the SGW-Ds in order to get periodic load statistics. By comparing the received load statistics with the SGW-D capability, the OF-ctr can easily get the load status of each SGW-D and therefore perform more efficient load balancing (i.e. based on the current load of SGW-Ds).

SGW data plane (SGW-D): represents an advanced OF switch that is able to encapsulate/decapsulate GTP packets. This switch applies the rules received from the OF-ctr. It is responsible for packet forwarding between the eNB and PGW. $\boldsymbol{e N B}$ : keeps the same radio functions specified by $3 \mathrm{GPP}$ standards. It is enabled with the OF protocol for the data 
forwarding through the S1 interface. Therefore, the data forwarding is based on instructions received from the OF-ctr. $\boldsymbol{P G W}$ : still has the same function as in the 3GPP standards.

The TEID values allocation in the SGW-C is performed once per session. These values remain invariant during moving the session from one SGW-D to another. Actually, when the SGW-C commands the OF-ctr to relocate a new SGW-D for a specific session, the OF-ctr will just update in the eNB the flow entry related this session with the IP address of the new SGW-D. Also, the SGW-C updates the SGW-D IP address in the PGW.

\section{B. Data Plane establishment procedure}

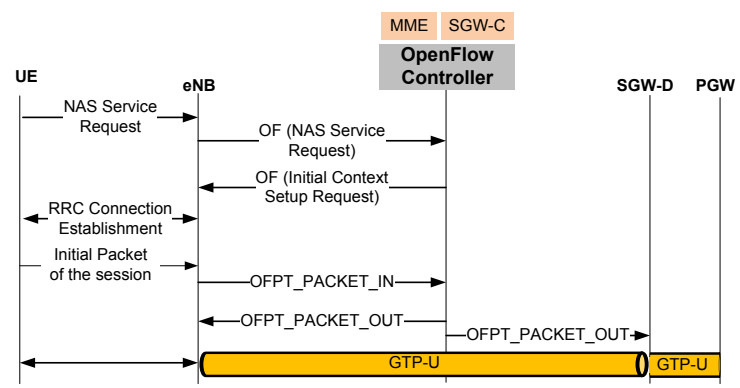

Fig. 3. Data plane establishment.

This procedure is required for each newly launched session. First, the UE sends to the MME the NAS Service Request message to get the authorization to establish the radio data bearer. The UE sends to the eNB the initial packet via the established radio data bearer. Then, the eNB checks its flow tables. As no flow entry exists for this initial packet, the eNB sends to the OF-ctr the packet header via the OFPT_PACKET_IN message. Also, the eNB includes in this message the eNB-TEID value for the downlink traffic in S1 interface. The OF-ctr analyzes the packet header to identify the source IP address, the destination IP address and the session type. The OF-ctr presents these information to the SGW-C. Based on the IP addresses and the load statistics collected by the OF-ctr, the SGW-C selects the adequate SGW-D. The session type enables the SGW-C to decide the appropriate QoS. For instance, if the packet belongs to VoIP session and the already selected SGW-D is overloaded, the OF-ctr decides to allocate another SGW-D with less load. The SGW-C sends back to the OF-ctr the SGW-D IP address, the SGW-TEID values and the QoS level. The OF-ctr creates a flow entry for the subsequent packets related to the same session and sends it to the eNB via the OFPT_PACKET_OUT message. The action field of this flow entry includes the SGW-D IP address and the SGW-TEID for the uplink traffic in the S1 interface. Similarly, the OF-ctr creates and sends to the SGW-D a flow entry related to this session via the OFPT_PACKET_OUT message. The action field of the flow entry includes the eNB IP address, the eNB-TEID, the SGW-TEID for the uplink traffic in the S1 interface, the PGW IP address, the PGW-TEID, and the SGW-TEID for the downlink traffic in S5 interface.
The OF-ctr decides the UE inactivity timer value and includes it each flow entry. Therefore, when the UE inactivity timer in the flow entry expires and no packet arrives, the eNB or the SGW-D just deletes this flow entry. Unlike the current LTE/EPC architecture, no further signaling is needed to release the access bearer.

\section{On-Demand Connectivity SeRvice}

In this section, we study two key aspects of the on-demand connectivity services namely resiliency and load balancing. First, we show that these aspects are not ensured with the current LTE/EPC architecture. Then, we demonstrate how the proposed architecture addresses both of these requirements.

\section{A. Resiliency}

Any network equipment failure in such scenario brings tremendous strain on network operators because it may lead to temporary service outage. Restoration procedures are crucial for reliable connectivity service in access networks.

1) Resiliency in 3GPP LTE/EPC architecture: The 3GPP restoration procedure [15] related to the $\mathrm{SGW}$ failure is depicted in Fig. 4. This failure can be detected by the MME or PGW via the incremented counter in the GPRS Tunnel Protocol (GTP) echo messages. Upon detecting the SGW 1 failure, the MME initiates the access bearer release procedure for active sessions that go through this failing SGW. Then, the MME assigns SGW 2 to the impacted users (i.e. idle and active users who were assigned to failing SGW) and triggers this new SGW to update all the impacted S5 bearers. Obviously, the MME informs the SGW 2 about the PGW address and the PGW-TEID value related to each impacted S5 bearer. The SGW 2 allocates new SGW-TEID values for the S5 bearers and sends them to the PGW. The MME should receive the Service Request message from the UE or Downlink Data Notification message from the SGW 2 to complete the restoration of active sessions.

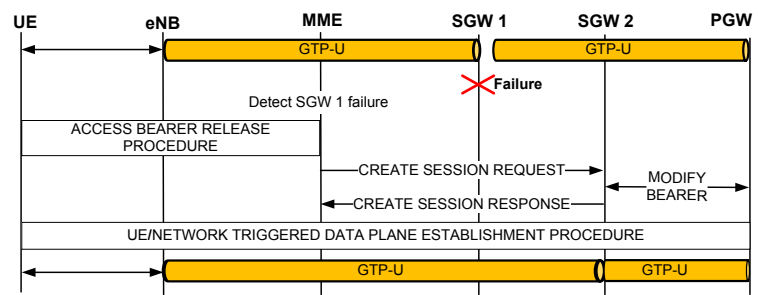

Fig. 4. 3GPP restoration procedure after a SGW failure

As we can see, the $3 \mathrm{GPP}$ restoration procedure related to the SGW failure is not transparent as it cuts off active sessions and waits till the UEs initiate the service request procedure again. Moreover, the session re-establishment of impacted users may generate significant amount of signaling because new GTP tunnels should be established. For instance, when the SGW relocation procedure takes place, new SGW-TEID values should be allocated by the target SGW and notified to the PGW and the eNB. The TEID allocation is a key function in the GTP tunnel establishment. We expect that separating such control function from the forwarding plane can enable fast failure recovery. 
2) Resiliency in OF-based LTE/EPC architecture: With our proposal, the SGW failure can be easily handled (see Fig. 5). As the OF-ctr and SGW-Ds exchange periodic Echo Request/Reply messages, the OF-ctr can detect any SGWD failure. Upon detecting the SGW-D 1 failure, the SGWC selects SGW-D 2 for the impacted sessions. The SGW-C updates the SGW-D IP address maintained in the PGW via the Modify Bearer Request. We propose to use the same interface to relay the communication between the SGW-C and PGW. As specified in our architecture, the SGW-TEID values for the downlink traffic on the S5 interface remain the same for the impacted sessions. After that, the OF-ctr updates the SGWD IP address in the eNB via the OFPT_MODIFY_STATE message. Here, we can see the advantage of centralizing the TEID allocation funtion related to SGWs. Indeed, the SGW$\mathrm{C}$ does not create new TEID values during the restoration procedure. The OF-ctr updates just flow entries in eNBs with the new SGW-D IP address. As the eNBs remain the same for each session, the eNB-TEID values for the downlink traffic in the S1 interface does not change. The OF-ctr inserts the new flow entries in the SGW-D 2 via the PACKET_OUT message.

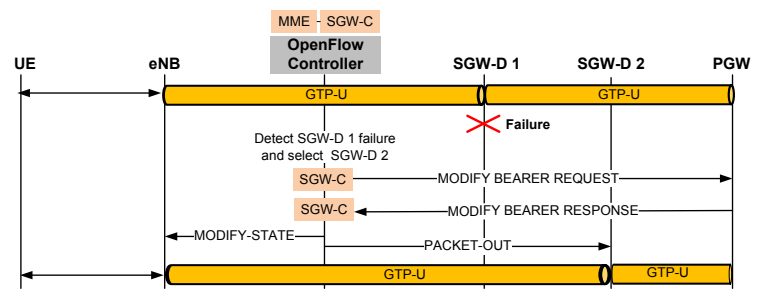

Fig. 5. Restoration procedure using an OpenFlow after a SGW failure.

\section{B. Load Balancing}

The Load balancing mechanism is required in the EPC architecture to spread the load across multiple network SGWs and PGWs, thereby preventing bottlenecks.

1) Load Balancing in 3GPP LTE/EPC architecture: SGW and PGW selection is performed by the MME and based on Weight Factors (WF) that are downloaded from the Domain Name Server (DNS) [13]. The WF is set according to the gateway capacity compared to the capacity of concurrent gateways. For instance, the SGW-WF is set according to the capacity of a SGW relative to other SGWs serving the same area. As the MME consider the SGW capacity before assigning the UE traffic to the appropriate SGW, we can say that it performs the proactive load balancing. Although this preventive approach, the SGW may experience periods of congestion as the current load balancing mechanism does not consider the SGW load in real-time. Indeed, an overload situation takes place when the packet arriving rate at the SGW is higher than the SGW service rate. Without no means to know the load of SGWs in real-time and the session type, the current MME keeps assigning users to the same SGW leading to bottlenecks.

2) Load Balancing in OF-based LTE/EPC architecture: Getting periodic statistics [16] about the SGW-D load is one of the advantages of implementing OpenFlow in the EPC architecture. These statistics are crucial for more efficient load balancing. For instance, based on real-time load statistics presented by the OF-ctr and the session type (i.e. determined from packet header), the SGW-C can balance the traffic between SGW-Ds leading to better traffic distribution. Unlike the 3GPP standards, our architecture can temporarily free the overloaded SGW by moving some sessions seamlessly to another SGW in the same domain.

\section{IMPLEMENTATION CHALLENGES}

To implement the proposed architecture, several challenges should be overcome, as listed next:

- The SGW control functions, such as the TEID allocation, should be first separated from the data forwarding plane. These control functions should run as applications on top of the OF controller. Similarly, the MME functions should be turned into applications that also run on the top of the OF controller. Likewise, the SGW and PGW selection functions should be shifted into another application.

- The OF controller should have a global vision of his domain topology and a real-time knowledge of the network equipment characteristics, such as the load. This is required for the SGW and PGW selection. We need thus to assess the implications from an implementation point of view, e.g., the trade-off between consistency and high availability.

- The SGW-C is likely to need a large database to store the required information about the networking state under its domain of control (such as active flow entries, allocated TEID values, etc.). Consequently, appropriate memory, IO, and CPU capabilities are required to store such information and to calculate the adequate handling for each session (e.g., decisions for routing, mobility, and QoS treatments).

- The OF protocol should be extended to transport the UE-MME exchanges transparently, e.g. the authentication exchanges. Moreover, the OF switch should be extended with the GTP encapsulation/decapsulation functions. For example, the current port data structure in the OF switch does not contain the GTP parameters, namely the destination and the source TEID values.

\section{Vi. Conclusion ANd Perspectives}

In this paper, we proposed an OpenFlow-based control plane for LTE/EPC architecture. Particularly, this architecture splits between the control and data forwarding planes related to the Serving Gateways (SGWs). The SGW control plane is centralized and uses the OpenFlow protocol to remotely manage the SGW data forwarding plane. This feature guarantees the on-demand connectivity service. We showed that the proposed architecture easily ensures the on-demand connectivity service even in critic situation such as network equipment failure and overload situations. Our next step consists in realizing a testbed to validate the resiliency and load balancing aspects in proposed OF-based LTE/EPC architecture. 


\section{ACKNOWLEDGMENT}

The work in this paper has been partially funded by the European Union Seventh Framework Programme through the Mobile Cloud Networking under grant agreement no. FP7ICT-318109. Further details about the project can be found in the website http://www.mobile-cloud-networking.eu/. To receive news, you can follow the twitter channel

@MobileCloudNets.

\section{REFERENCES}

[1] "Cisco visual networking index: Global mobile data traffic forecast update, 2012-17," 6 Feb 2013.

[2] Tellabs, "Tellabs "end of profit" study executive summary," Tech. Rep., January 2011.

[3] U. Paul, A. Subramanian, M. Buddhikot, and S. Das, "Understanding traffic dynamics in cellular data networks," in INFOCOM, 2011 Proceedings IEEE, april 2011.

[4] I. Sato, A. Bouabdallah, and X. Lagrange, "Improving lte/epc signaling for sporadic data with a control-plane based transmission procedure," in 14th International Symposium on Wireless Personal Multimedia Communications (WPMC), oct. 2011.

[5] C. Bernardos, J. Zunniga, and A. Reznik, "Towards flat and distributed mobility management: A 3GPP evolved network design," in IEEE International Conference on Communications (ICC), Jun 2012.

[6] I. Widjaja, P. Bosch, and H. La Roche, "Comparison of mme signaling loads for long-term-evolution architectures," in IEEE 70th Vehicular Technology Conference Fall (VTC 2009-Fall), sept. 2009.

[7] C. Xue, J. Luo, R. Halfmann, E. Schulz, and C. Hartmann, "Inter gw load balancing for next generation mobile networks with flat architecture," in IEEE 69th Vehicular Technology Conference, April 2009, pp. 1-5.

[8] J. Kempf, B. Johansson, S. Pettersson, H. Luning, and T. Nilsson, "Moving the mobile evolved packet core to the cloud," in IEEE 8th International Conference on Wireless and Mobile Computing, Networking and Communications (WiMob), oct. 2012.

[9] MEVICO, "D2.2 Architectural EPC extensions for supporting heterogeneous mobility schemes," Tech. Rep., Jan. 2013.

[10] X. Jiny, L.-E. Li, V. Laurent, and R. Jennifer, "Cellsdn: Software-defined cellular core networks," in open net summit, 2013.

[11] "Software-defined networking: The new norm for networks," Open Networking Foundation (ONF), White Paper, Apr. 2012.

[12] M.-K. Shin, K.-H. Nam, and H.-J. Kim, "Software-defined networking $(\mathrm{sdn})$ : A reference architecture and open apis," in 2012 International Conference on ICT Convergence (ICTC), Oct 2012, pp. 360-361.

[13] 3GPP, "General Packet Radio Service (GPRS) enhancements for Evolved Universal Terrestrial Radio Access Network (E-UTRAN) access," 3rd Generation Partnership Project (3GPP), TS 23.401, Jun. 2011.

[14] 3GPP, "General Packet Radio Service (GPRS); GPRS Tunnelling Protocol (GTP) across the Gn and Gp interface," 3rd Generation Partnership Project (3GPP), TS 29.060, Mar. 2013.

[15] 3GPP, "Restoration procedures," 3rd Generation Partnership Project (3GPP), TS 23.007, Dec. 2012.

[16] Open Networking Foundation, "OpenFlow Switch Specification, version 1.3.1," September 62012. 